\title{
Laparoscopic approach to ovarian pathology in children and adolescents
}

\begin{abstract}
Laparoscopic procedures are constantly finding wider pplication in general and pediatric surgery. The female child and adolescent presenting with suspected acute or chronic ovarian pathology may be an ideal candidate for laparoscopic surgery. From March 1992 to December 1993, six girls aged 7 to 18 years underwent seven aparoscopic operations for ovarian pathology at Childrens Hospital Los Angeles. Clinical presentations include 1 virilization with primary amenorrhea (1), precocious puberty (1), malignant dysgerminoma (second look) (1), and abdominal pain (3). Postoperative diagnoses were dysgerrinoma (1), bilateral gonadoblastoma in a $46 \mathrm{XY}$ chromosomal phenotypic female with dysgenetic gonads (1), neeative biopsy (1), serous cystadenoma with acute torsion (1), ovarian cyst (1), and ovarian torsion (1). Operations performed were unilateral salpingo-oophorectomy (1), unilateral salpingo-oophorectomy with contralateral biopsy (2), bilateral salpingo-oophorectomy (1), diagnostic biopsy (2), and ovarian cystectomy with oophoropexy (1). There were no operative complications. One patient underwent two operations, a biopsy followed by unilater:l salpingo-oophorectomy. Only one patient required a mini-laparotomy for delivering a large tumor mass. Mean operative time was $138 \mathrm{~min}$, mean hospital stay 2.0 days. We encountered two malignant cases, which were de finitively treated by laparoscopic resection. These two patients remained free of disease 12 to 18 months later. The laparoscopic approach to ovarian pathology in children and adolescents is an effective method for diagnosis as well as defir itive therapy. Our initial experience is presented followec by recommendations for clinical practice.
\end{abstract}

Key wol:ds Pediatric laparoscopy $\cdot$ Ovarian pathology

S. Kanno - S. Emil - J. B. Atkinson (

Division of Pediatric Surgery, Childrens Hospital Los Angeles,

Los Angeles, California, 4650 Sunset Boulevard, Los Angeles

CA 90027, USA

L. Takeuchi

Division of Pathology, Childrens Hospital Los Angeles,

4650 Sunset Boulevard, Los Angeles, CA 90027, USA

\section{Introduction}

Laparoscopy for adult gynecologic pathology was commonplace long before the recent revolution in laparoscopic surgery. In 1973, Gans and Berci reported their experience with five pediatric patients who were successfully explored and biopsied laparoscopically for intersex problems, gonadal dysplasia, and precocious puberty [4]. Shortly afterward, Cognat et al. reported 60 laparoscopic cases in children as young as 6 months for a variety of gynecological problems [2]. In 1991, Shalev succeeded in performing laparoscopic detorsion of an adnexa in a child [15] and in 1992, Heloury et al.'s series of laparoscopies for pediatric adnexal pathology was reported from France [6]. Recently, Tan et al. presented two children who successfully underwent laparoscopic oophoropexy for pelvic malignancies [17]. The pediatric surgical experience with laparoscopy for suspected or diagnosed ovarian pathology continues to develop.

\section{Materials and methods}

From March 1992 to December 1993, six girls aged 7 to 18 years underwent seven laparoscopic operations for ovarian pathology at Childrens Hospital Los Angeles. Their medical records were reviewed retrospectively. All patients underwent general endotracheal anesthesia and were placed in lithotomy position. A Foley catheter drained the bladder. In older, sexually active girls, the cervix was exposed with a small vaginal speculum and grasped with a uterine tenaculum. A sound was then inserted into the uterine cavity to allow manipulation of the uterus during laparoscopy. Figure 1 illustrates the operative set-up.

Using an umbilical cutdown technique, an infraumbilical location was selected and the skin, fascia, and peritoneum were exposed and entered. A blunt $10-\mathrm{mm}$ trocar was then introduced and used to insufflate the abdomen. Adequate visualization can usually be achieved with $10 \mathrm{mmHg}$ insufflation pressure in small infants; higher pressures may be used in older children up to $15 \mathrm{mmHg}$. The laparoscope was inserted and the abdomen inspected for any abnormalities. Usually two additional 5 - or 10-mm trocars were inserted under direct visualization in the right and left anterior axillary lines, just below the umbilical trocar (Fig. 2). Exact port placement was adjusted according to the size and configuration of each patient. These were 


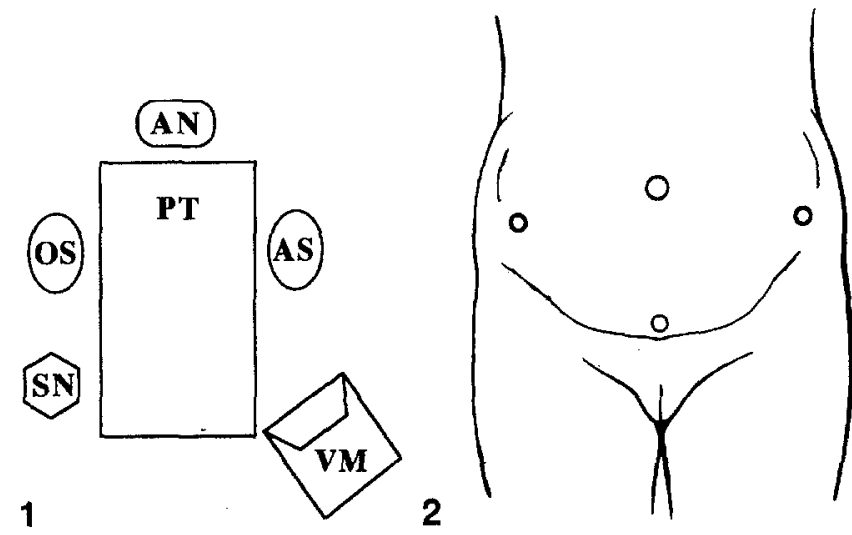

Fig. 1 Operating room set-up; OS: Operating Surgeon, AS: Assistant Surgeon, PT: Patient, AN: Anesthesiologist, SN: Scrub Nurse, VM: Video Monitor

Fig. 2 Cannula position

used to introduce appropriate instruments as required in each case. If additional access was needed, a $5-\mathrm{mm}$ port was added in the suprapubic area. After the abdominal cavity was inspected, the patient was placed in Trendelenburg position to begin the operative procedure.

\section{Case reports}

Case 1. An 18-year-old female presented with primary amenorrhea virilization, poor breast development, and delayed puberty. Elevated levels of follicle-stimulating hormone $76 \mathrm{mIU} / \mathrm{ml}$ (normal 2-9) luteinizing hormone $25 \mathrm{mIU} / \mathrm{ml}(0.2-9)$, and testosterone $281 \mathrm{ng} / \mathrm{dl}$ $(10-55)$ were found in addition to $46 \mathrm{XY}$ chromosomes. Pelvic ultrasound (US) showed no uterine or ovarian abnormalities. A plain abdominal X-ray film revealed small calcifications in the right pelvis. During laparoscopy, both gonads showed areas of patchy abnormalities. The abdomen was free of ascites or metastatic lesions. Biopsies with frozen section (FS) revealed malignant tumor in both ovaries but the tumors were small and the margins clear, so laparoscopic bilateral salpingo-oophorectomy was carried out. The final diagnosis was bilateral gonadoblastoma in a $46 \mathrm{XY}$ chromosomal phenotypic female with dysgenetic gonads. There were no postoperative complications. She remains disease-free 1.5 years later.

Case 2. A 7-year-old child presented with precocious puberty and elevated beta- human chorionic gonadotropin $378 \mathrm{mIU} / \mathrm{ml}$ (normal $<2.0$ ). An abdominal radiograph revealed calcification and abdominal US showed an enlarged right ovary $(4 \times 3 \mathrm{~cm})$ containing a small, hyperechoic focus at its upper margin. Surgery was performed to evaluate the right ovarian enlargement. Laparoscopic biopsy of both ovaries revealed an uncertain FS diagnosis of granulosa cell tumor of the right ovary. Pelvic fluid was aspirated for cytology and the operation was terminated. A right salpingo-oophorectomy was accomplished laparoscopically 2 days after the first surgery when permanent section revealed a dysgerminoma. The patient had no complications and was discharged the day following her second operation. She is disease-free with follow-up extending over 1 year.

Case 3. A 9-year-old girl had undergone an open right salpingooophorectomy for dysgerminoma. At the time of her open operation, a left ovarian biopsy also showed involvement with tumor (stage IB). It was elected at the time to spare the left ovary and treat the patient with chemotherapy. Four months after her primary surgery, the patient underwent laparoscopy for a second look and repeat biopsy. There was no tumor present clinically or pathologically. The patient was discharged the next day without complications and required no further therapy.
Case 4. A 14-year-old girl presented with 2 days of severe lower abdominal pain and vomiting without fever, leukocytosis, or anemia. US revealed a $9 \times 7-\mathrm{cm}$ left lower quadrant mass suspicious for an ovarian teratoma with possible torsion. Laparoscopy should a large, heterogeneous mass associated with the left ovary in addition to complete torsion and infarction. A left salpingo-oophorectomy was completed laparoscopically, but a 4-cm mini-laparotomy centered at the suprapubic trocar site was necessary to deliver the mass. A right ovarian wedge biopsy was also done. The pathologic diagnosis was a left serous cystadenoma with hemorrhagic necrosis. The patient tolerated a regular diet on the 2 nd postoperative day and was discharged on the 4 th day.

Case 5. A 13-year-old girl presented with left lower abdominal pain of several weeks' duration. Clinical data were within normal range. An abdominal radiograph was unremarkable and US revealed a solid $6 \times 4$ $\mathrm{cm}$ mass in the left lower quadrant. A left salpingo-oophorectomy was completed laparoscopically. However, in order to deliver the large mass intact, the infraumbilical incision had to be extended $1 \mathrm{~cm}$ laterally in each direction. The anterior and posterior rectus sheaths of this extended incision were closed with 2-0 Vicryl sutures. A right ovarian wedge biopsy was also done. Pathologic examination showed a torsed, edematous, necrotic ovary uninvolved by tumor. The patient was discharged on the 2 nd postoperative day without complications.

Case 6. An 11-year-old girl presented with abdominal pain for several weeks, 6 years status post-mini-laparotomy for excisional biopsy of bilateral ovarian cysts. US demonstrated a cystic pelvic mass. Laparoscopy showed a large cystic mass occupying approximately $80 \%$ of the left ovary, located behind the uterus and suspicious for torsion. The mass was aspirated and then excised within a specimen bag laparoscopically. A left oophoropexy was performed to prevent possible torsion. Pathologic examination revealed a simple, benign cyst and the patient was discharged on the 2 nd postoperative day without complications.

\section{Results}

The cases are summarized in Table 1. Table 2 lists pertinent statistics for this series of patients. Mean operative time was $138 \mathrm{~min}$ and there were no operative complications. Only one patient required a mini-laparotomy for delivering a large tumor mass. The mean hospital stay was 2.0 days. We encountered two malignant cases, which were definitively treated by laparoscopic resection. These two children remained free of disease 12 to 18 months later.

\section{Discussion}

The dawn of modern laparoscopic surgery was heralded by the wide acceptance of laparoscopic cholecystectomy by surgeons and patients alike. Since then, laparoscopy has grown to include many of the general surgical procedures traditionally performed by open approaches. These methods are becoming increasingly popular in pediatric surgery: laparoscopic cholecystectomy has also been successfully performed in infants and children $[8,11,16]$. A recent symposium highlighted the role of laparoscopy in fundoplication, splenectomy, cholangiography, appendectomy, and other procedures in the pediatric age group [7].

As early as 1973, Gans and Berci reported their experience with five patients who were successfully ex- 
Table 1 Experience with laparoscopy for ovarian pathology in children and adolescents

\begin{tabular}{lllll}
\hline $\begin{array}{l}\text { Case } \\
\text { no. }\end{array}$ & $\begin{array}{l}\text { Age } \\
\text { (years) }\end{array}$ & Indication & Procedure & Diagnosis \\
\hline 1 & 18 & Primary amenorrhea, virilization & Bilateral salpingo-oophorectomy & Gonadoblastoma/dysgerminoma, 46XY \\
2 & 7 & Precocious puberty & Bilateral biopsy & Dysgerminoma \\
& 7 & Dysgerminoma & L. Salpingo-oophorectomy & Dysgerminoma \\
3 & 9 & Second look post-chemotherapy & L. Biopsy & No recurrent tumor \\
4 & 14 & Acute abdominal pain & L. salpingo-oophorectomy; & Serous cystadenoma with hemorrhagic \\
& 13 & Subacute abdominal pain & L. wedge biopsy & Tocrosis
\end{tabular}

plored and biopsied laparoscopically for intersex problems, gonada dysplasia, and precocious puberty [4]. Soon after, Cognat et al. reported 60 cases in children as young as 6 months managed laparoscopically for a variety of gynecologic al problems [2]. These authors were able to perform accurate explorations, biopsies, fluid aspirations, and other simple procedures using the equipment available at that time. More recently, successful laparoscopic detorsion of adnexa in a child [15], laparoscopic pre-radiation oophoropexy ior pelvic malignancies [17], as well as a larger French series of laparoscopies for pediatric adnexal pathology [6] were reported. Moir has reviewed the technical details and indications for laparoscopy in the pediatric female patient [10].

Laparoscopy for ovarian pathology in children may be best approached by dividing patients into two groups depending on presentation: acute or subacute abdominal pain versus elective indications. We recommend that a preoperative complete blood count and pelvic US be obtained in all patients in whom ovarian pathology is suspect $\mathrm{d}$. In addition, those with an adnexal mass should have preoperative tumor markers documented. Additional imaging studies may be obtained as indicated. Elective indications in our series were virilization with primary amenor hea, precocious puberty, and a second-look procedure. A.dditional indications include gonadectomy in patients with Turner's syndrome or other intersex problems, endome riosis, benign teratoma, or chronic pelvic pain. These diseases can all be diagnosed and definitively treated laparoscopically.

Our initial experience at a large pediatric hospital points to a promising role for laparoscopy in the management of adnexal pathology in children and adolescents. Laparoscopy correctly identified the adnexal pathology in each

Table 2 ?ediatric laparoscopy for ovarian pathology

\begin{tabular}{lcl}
\hline Age rang: & $7-18$ & years (mean 11.7) \\
Operating; time & $75-205$ & min (mean 138) \\
Complication rate & 0 & $\%$ \\
Mini-laparotomy & 1 & case \\
Postoperative hospital stay & $1-4$ & days (mean 2.0) \\
Malignan: cases & 2 & \\
\hline
\end{tabular}

case and accomplished the surgical goals without intra- or postoperative complications. One patient required a minilaparotomy and one required extension of a trocar incision for delivering large tumor masses. Biopsy, unilateral and bilateral salpingo-oophorectomy, cyst aspiration, cystectomy, and oophoropexy were all performed successfully with the available instrumentation. Although the number is small, the procedure appears to be superior with respect to postoperative pain and hospital stay when compared to laparotomy for similar indications. Operative time is longer, but we expect this to improve with experience as has been the case with other laparoscopic procedures.

The young female with acute or subacute abdominal pain may be the ideal candidate for laparoscopy. Traditionally, this group has had the highest percentage of erroneous preoperative diagnoses, as well as unnecessary operations [10]. A surgeon who finds a normal appendix through a right lower quadrant incision has only two options: to extend the incision for adequate exploration of the abdomen and adnexa or close the incision and observe the patient. Each of these choices may have negative repercussions for the patient and may increase the cost and prolong the hospital stay. A better approach is to explore the abdomen and pelvis laparoscopically and directly diagnose the pathology, most of which can also be definitively treated laparoscopically. In cases of ovarian malignancy, it is impossible to inspect the diaphragm for metastases with accuracy through a Pfannensteil incision. Appendectomy, adnexal detorsion, oophoropexy, aspiration and/or excision of ovarian cysts, biopsies, evacuation of an ectopic pregnancy, and drainage of a tubo-ovarian abscess are all procedures that can be accomplished laparoscopically, although simultaneous gynecologic expertise would be best sought for some.

The role of laparoscopy in the management of an adnexal mass suspicious for malignancy is not well defined. Although the risk of "spilling" cancer cells in the peritoneal cavity remains theoretical, adult gynecologists still discourage laparoscopy for any patient with clinical, sonographic, or other imaging characteristics suspicious for ovarian malignancy $[5,9,12,13]$. Two previous large series from our institution identified malignancy in $16 \%-25 \%$ of all ovarian cysts and tumors in children [3, 18]. More 


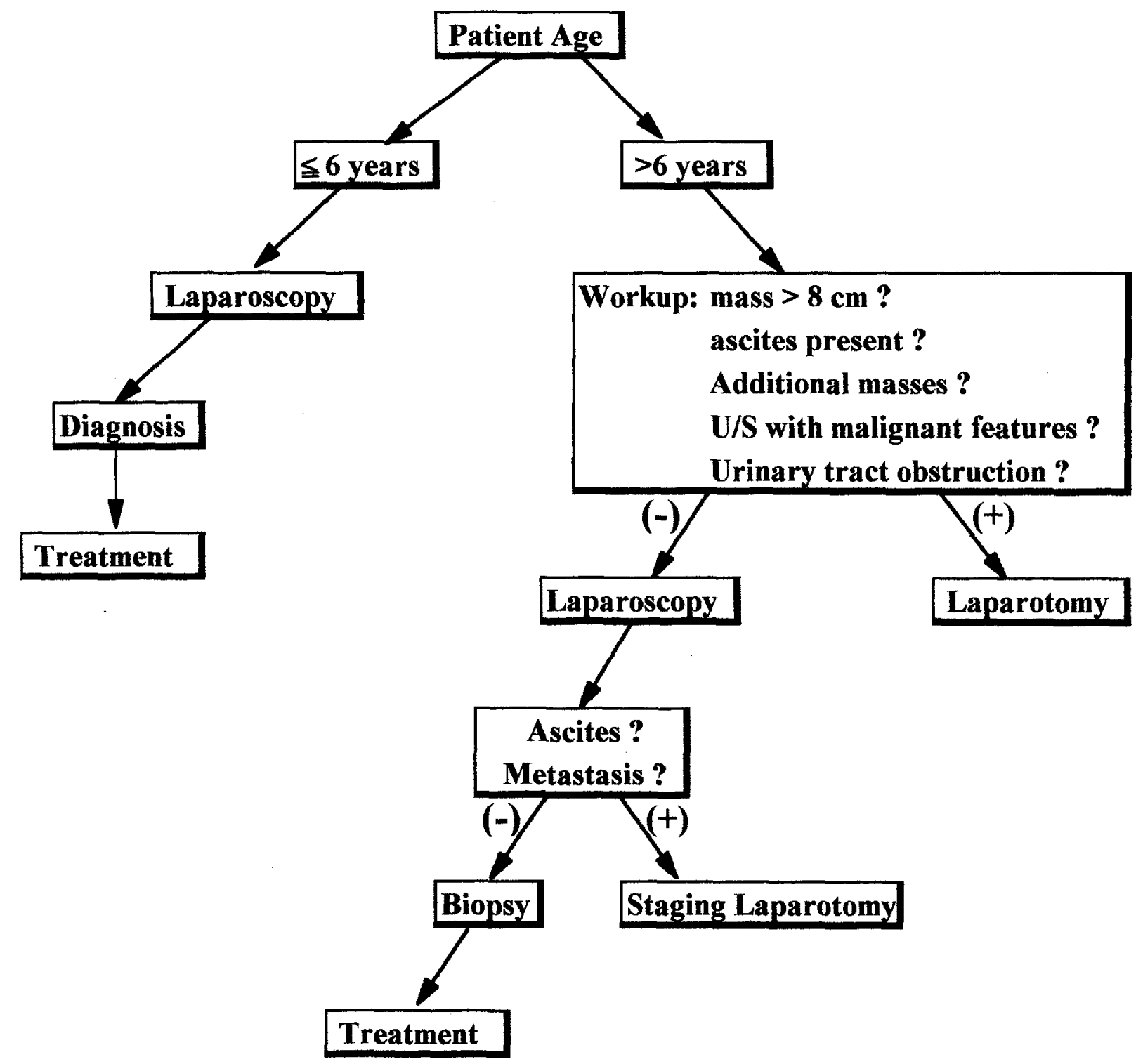

Fig. 3 Approach to adnexal masses in children

recently, Brown et al. showed, in a review of 91 cases, a malignancy rate of $2.9 \%$ for children under 8 years of age that jumped to $33 \%$ for older children [1]. A recent review of 61 patients from our institution over a decade revealed a malignancy rate of $7 \%$ in girls under 6 years of age versus $34 \%$ in older children.

The size of the mass is also an issue. In adult patients, ovarian masses larger than $10 \mathrm{~cm}$ on US carry a $72 \%$ chance of malignancy versus $1 \%$ and $11 \%$ for masses less than $5 \mathrm{~cm}$ and $5-10 \mathrm{~cm}$, respectively $[5,12,13]$. A mass larger than $8 \mathrm{~cm}$ is also very likely to require a minilaparotomy for removal from the abdominal cavity. Although a morcellator and sac can be used and have been reported to produce adequate tissue for pathologic study [14], this is not a well-established procedure for accurate evaluation of malignant specimens. In our practice, body weight less than $5 \mathrm{~kg}$ is still a contraindication to laparoscopy.
Based on our present knowledge, we recommend the following approach to an adnexal mass in girls as outlined in this algorithm (Fig. 3). If the child is under 6 years of age, laparoscopic management is recommended for diagnosis and definitive therapy. In an older child, the preoperative work-up should heavily influence the surgical decision. A mass smaller than $8 \mathrm{~cm}$, lacking malignant features on US, and not associated with ascites, other abdominal masses, or urinary obstruction may be approached laparoscopically. On exploration, fluid should always be collected for cytology. Gross evidence of metastasis or ascites should dictate conversion to a formal staging laparotomy. If no such evidence exists, biopsies with frozen section can guide adequate excision. Cystic lesions may be decompressed within a specimen bag, if there is a low index of suspicion for tumor. We believe that adequate margins of resection can be achieved laparoscopically in uncomplicated cases of malignancy. 
In conclusion, acute, subacute, and chronic ovarian pathology in girls can be effectively diagnosed and treated laparos zopically by the pediatric surgeon, with gynecologic assistarce as needed. The approach to adnexal masses can be guiced by age of the patient, preoperative work-up, and operative findings. Solid tumors larger than $8 \mathrm{~cm}$ may require laparotomy for extraction. Cystic tumors may be resecte:1, bagged, and then aspirated for laparoscopic extraction. If there is no evidence of metastatic disease, resecticn for malignant disease can be accomplished by laparoscopy without compromise of the surgical margin, although size may be a limitation. The rewards should be improved morbidity, decreased hospital stay, decreased cost, and improved cosmesis, although they remain to be demonstrated in larger studies.

\section{Referer ces}

1. Brotin MF, Hebra A, McGeehin K, et al (1993) Ovarian masses in chilcren; a review of 91 cases of malignant and benign masses. J Pei.iatr Surg 28: 930-932

2. Cognat M, Papathanassiou Z, Gomel V (1974) Laparoscopy in infar ts and adolescents. J Reprod Med 13: 11-12

3. Ehren IM, Mahour GH, Isaacs H Jr (1984) Benign and malignant ovar an tumors in children and adolescents; a review of 63 cases. Am I Surg 147: 339-344

4. Gans SL, Berci G (1973) Peritoneoscopy in infants and children. J Pediatr Surg 8: 399-405

5. Granberg S, Wikland M, Jansson I (1989) Macroscopic characterizaticn of ovarian tumors and the relation to the histological diagnosis; criteria to be used for ultrasound evaluation. Gynecol Oncol 35: 139-144
6. Héloury Y, Guiberteau V, Sagot P, et al (1993) Laparoscopy in adnexal pathology in the child; a study of 28 cases. Eur $J$ Pediatr Surg 3: $75-78$

7. Holcomb GW III, Georgeson K, Lobe TE, et al (1994) Pediatric laparoscopy and thoracoscopy. Contemporary Surg 44: 183-199

8. Holcomb GW III, Naffis D (1994) Laparoscopic cholecystectomy in infants. J Pediatr Surg 29: 86-87

9. Hulka JF, Parker WH, Surrey MW, et al (1992) Management of ovarian masses; AAGL 1990 survey. J Reprod Med 37: 599-602

10. Moir CR (1994) Laparoscopy for the female patient. In: Holcomb GW III (ed): Pediatric endoscopic surgery. Appleton \& Lange, Norwalk, Conn, pp 51-58

11. Newman KD, Marmon LM, Attorri R, et al (1991) Laparoscopic cholecystectomy in pediatric patients. J Pediatr Surg 26: $1184-1185$

12. Parker WH (1992) Management of adnexal masses by operative laparoscopy; selection criteria. J Reprod Med 37: 603-606

13. Rulin MC, Preston AL (1987) Adnexal masses in postmenopausal women. Obstet Gynecol 70: 578-581

14. Schropp KP, Lobe TE (1994) Tissue extraction. In: Lobe TE, Schropp KP (eds) Pediatric laparoscopy and thoracoscopy. WB Saunders, Philadelphia, pp 52-58

15. Shalev E, Mann S, Romano S, et al (1991) Laparoscopic detorsion of adnexa in childhood; a case report. J Pediatr Surg 26: $1193-1194$

16. Sigman HH, Laberge JM, Croitoru D, et al (1991) Laparoscopic cholecystectomy; a treatment option for gallbladder disease in children. J Pediatr Surg 26: 1181-1183

17. Tan HL, Scorpio RJ, Hutson JM, et al (1993) Laparoscopic ovariopexy for paediatric pelvic malignancies. Pediatr Surg Int 8: $379-381$

18. Towne BH, Mahour GH, Woolley MM, et al (1975) Ovarian cysts and tumors in infancy and childhood. J Pediatr Surg 10: 311-320 